\title{
APPLICATION OF WSN IN CROCKERY MANUFACTURING INDUSTRY
}

\author{
Fareeha Zafar ${ }^{1}$, Azhar Hameed ${ }^{2}$, Muhammad Masood lqbal ${ }^{3}$ \\ ${ }^{1}$ Fareeha Zafar(Dr. ) Government College University Lahore, Department of Computer \\ science, Pakistan,Dr.f.zafar@gcu.edu.pk \\ ${ }^{2}$ Azhar Hameed (M.Phil. Scholar), Government College University Lahore, Pakistan, \\ muhammad.azharhameed@gmail.com \\ ${ }^{3}$ Muhammad Masood lqbal (M.Phil. Scholar), Government College University Lahore, Pakistan, \\ masood.iqbal.pak@gmail.com
}

\begin{abstract}
This Paper illustrates the usage of WSN in Crockery manufacturing unit. The paper has aimed to improve the production process with the help of Wireless Sensors in such a way that not only the system will be fault tolerant but it shall also be measured against the financial gains within the industry after inclusion of such sensors within the production unit. The research has focused to encounter slenderest negligence by the human resource that can result in loss of any produced item. The methodology explained will also provide mental relief to the human resource worker who is required to be at surveillance of produced items at all times during the manufacturing process in order to save the item from overheating, that may result in item loss. Another important feature of this research is to present WSN in such a way that the machinery used for Crockery production will be complete automatic i.e. without human interference process, which has not been practiced previously in this domain. The manufacturing unit of crockery is based in Pakistan and all the data and information presented in paper is taken from there.
\end{abstract}

Keywords: wireless sensor networks, crockery manufacturing, fault tolerance

\section{INTRODUCTION}

Wireless sensor networks hold the promise of many new applications in the area of monitoring and control. Examples include target tracking, intrusion detection, wildlife habitat monitoring, climate control, and disaster management.

In industry, WSNs can be used to monitor manufacturing process and the condition of manufacturing equipment. WSN are used in wide range of applications in the industrial domain, eliminates the requirement of human presence in various places including dangerous areas to obtain sensory information and actuation control. The paper will describe the use of WSN in a Local Crockery Manufacturing Unit in Pakistan. The objective of this research is to provide ease in manufacturing process and eliminates the chances of financial 
loss that can be occurred without the use of WSN. The proposed model will eliminate the chances of items from being spoiled due to overheating that will eventually results in financial gain. It eliminates the need of a well-trained worker, who has a complete command on the manufacturing unit, knows very well that when to open the dyes. Because all these tricky things are done by the Wireless Sensor Network, provides mental relief to the worker who has to keep his eyes all the time on the unit, be attentive in order to save the item from overheating.

Wireless sensor network (WSN) is a network of micro electro-mechanical systems (MEMS) [1] called "sensor devices" deployed to gather sensory information from an area of interest. Sensor devices (nodes) have the ability to sense, process, and communicate data. These devices typically have limited computing power and are designed to operate on batteries. Initially, these sensor devices were used in military applications [2]. Industrial Wireless Sensor Networks (IWSN) are an emerging class of WSN that faces specific constraints linked to the particularities of the industrial production. In these terms, IWSNs faces several challenges such as the reliability and robustness in harsh environments, as well as the ability to properly execute and achieve the goal in parallel with all the other industrial processes. Furthermore, IWSN solutions should be versatile, simple to use and install, long lifetime, low-cost devices, indeed, the combination of requirements hard to meet.

\section{LITERATURE REVIEW}

An earlier research that focused on preventive equipment maintenance, in which vibration signatures are gathered to predict equipment failure. They analyzed the application of vibration analysis for equipment health monitoring in a central utility support building at a semiconductor fabrication plant that houses machinery to produce pure water, handle gases and process waste water for fabrication lines. Furthermore, they deployed the same sensor network on an oil tanker in order to monitor the onboard machinery. In the end, they discuss design guidelines for an ideal platform, industrial applications, a study of the impact of the platform on the architecture, the comparison of two aforementioned deployments and a demonstration of application return on investment. [5]

In another application, a wireless network system developed for a team of underwater collaborative autonomous agents that are capable of locating and repairing scale formations in tanks and pipes within inaccessible environments. They described in detail ad-hoc network hardware used in their deployments that comprises the $\mathrm{pH}$, proximity, pressure sensors and repair actuator. Furthermore, they described the communication protocol and sensor/actuator feedback loop algorithms implemented on the nodes. [6]

Another work that focused on WSN for pipeline monitoring is [7], which described the WSN Applications of Industrial Wireless Sensor Networks 15 whose aim is to detect, localize and quantify bursts, leaks and other anomalies (blockages or malfunctioning control valves) in water transmission pipelines. The research reported the results, experiences from real deployment, provided algorithms for detecting and localizing the exact position of leaks that is tested in laboratory conditions. The system presented in this work is also used for monitoring water quality in transmission, distribution water systems and water level in sewer collectors. In this context, the work can be classified as the process evaluation group of works (Section 1.5.2.1) as well.

Another WSN for machinery condition-based maintenance, they presented design requirements, limitations and guidelines for this type of WSN applications. Furthermore, they implemented their condition monitoring system in Heating \& Air Conditioning Plant in Automation and Robotics Research Institute in University of Texas. [8]

Another work proposed the use of accelerometer based monitoring of machine vibrations, tackled the problem of predictive maintenance and condition-based monitoring of factory machinery in general. They demonstrated a linear relationship between surface finish, tool wear and machine vibrations thus proving the usability of proposed system in equipment monitoring. [9]

\section{RESEARCH CONTEXT}

The research is based on a local crockery Manufacturing unit at Gujranwala (Pakistan). Gujranwala is well known for crockery manufacturing and considered as crockery manufacturing hub in Pakistan.

The Crockery manufacturing unit consists of Automatic Compression Molding machine with Electro Hydraulic Press control system, Integral power pack and motor control equipment together with temperature controller. The manufacturing process is completed in two phases and each phase has several steps. 


\subsection{Phase I}

\subsubsection{First Step}

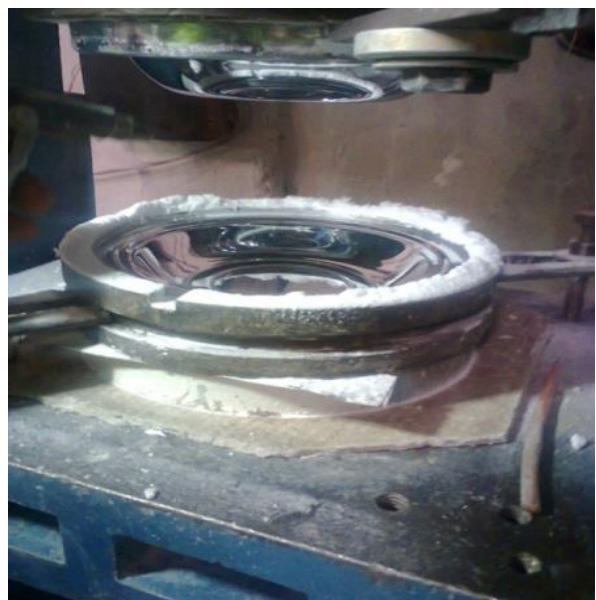

Figure 1

A pair of dyes is Fixed on the molding machine. The upper part of the machine is fixed while the lower one is moveable.

\subsubsection{Second Step}

\subsubsection{Third Step}

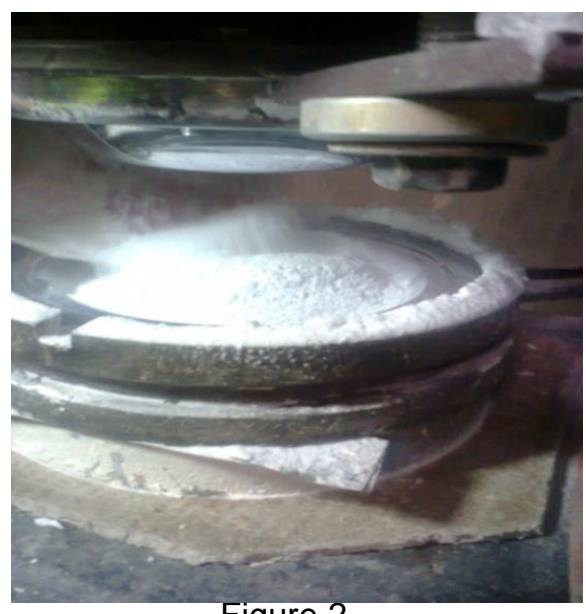

Figure 2

Lower part is filled with raw material.

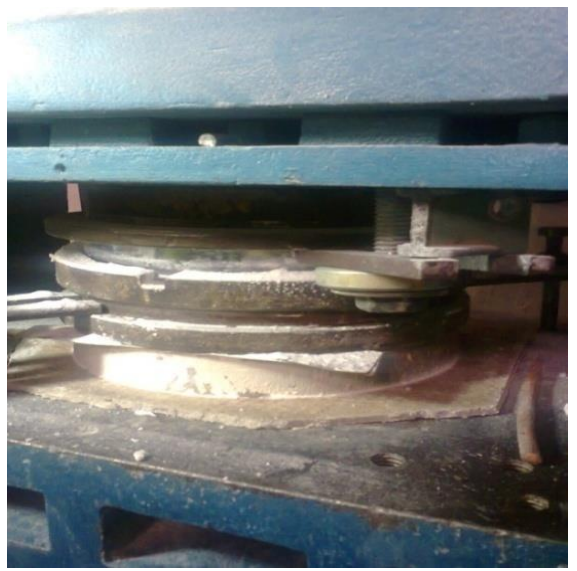

Figure 3

A worker pulls the gear of the Hydraulic part and as a result the Moveable part of the dye moves towards upward and joins the fixed part. 


\subsubsection{Fourth Step}

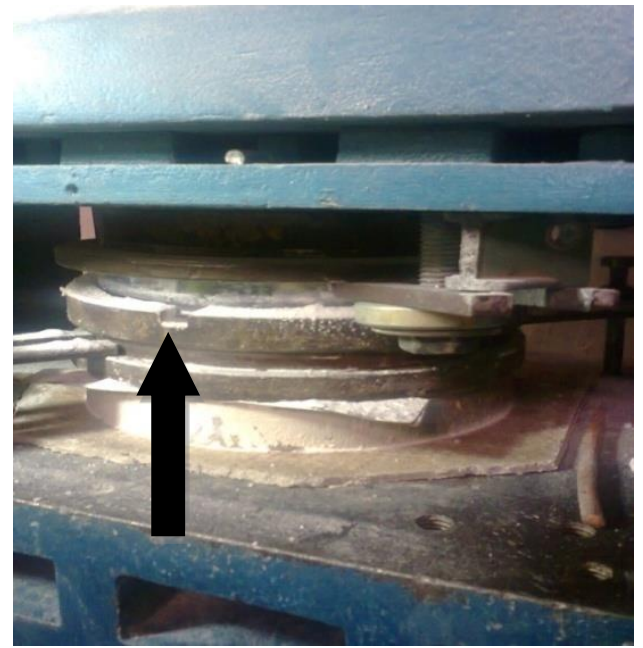

Figure 4

Now it is heated at a specific temperature until a small amount of material comes out from the little spillways.

\subsubsection{Crucial Moment}

This is a crucial moment, when the material starts to come out, worker has to push forward the gear immediately in order to disjoin both parts of dyes immediately, otherwise the temperature will spoil the item.

\subsection{Phase II}

3.2.1 Step 1

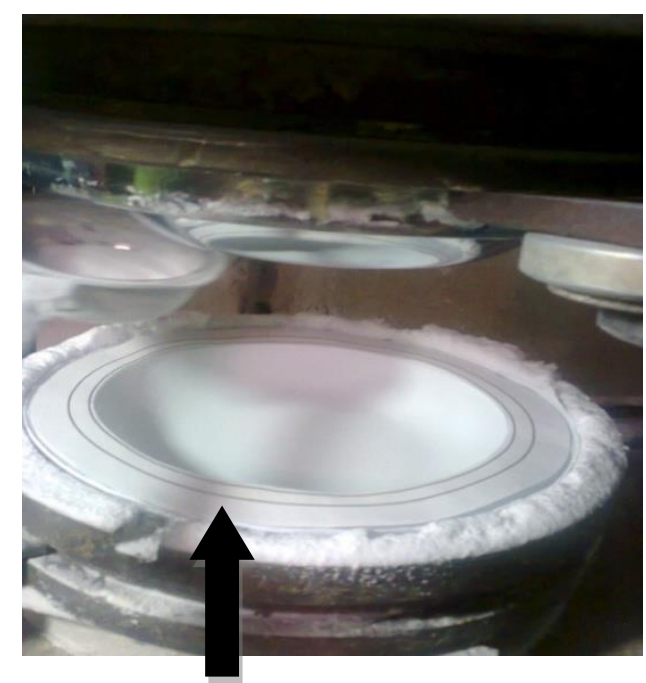

Figure 5

In this step, the foil of overlay is placed with partially cured piece. 


\subsubsection{Step 2}

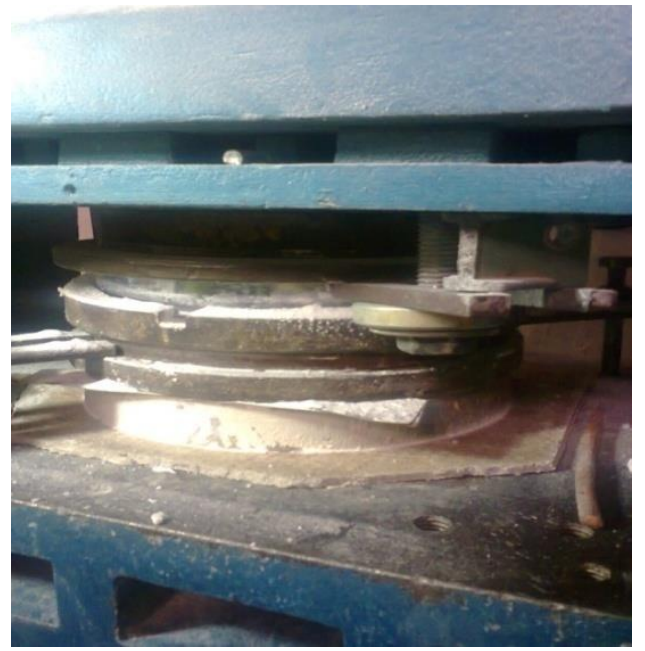

Figure 6

Again Moveable part joins the fixed one

\subsubsection{Crucial Moment}

Now again it is heated at specific temperature until the material again comes out. This is again a crucial point in manufacturing process. Disjoin the lower and upper part of the dye immediately; otherwise the temperature will spoil the item.

\subsection{Final Product}

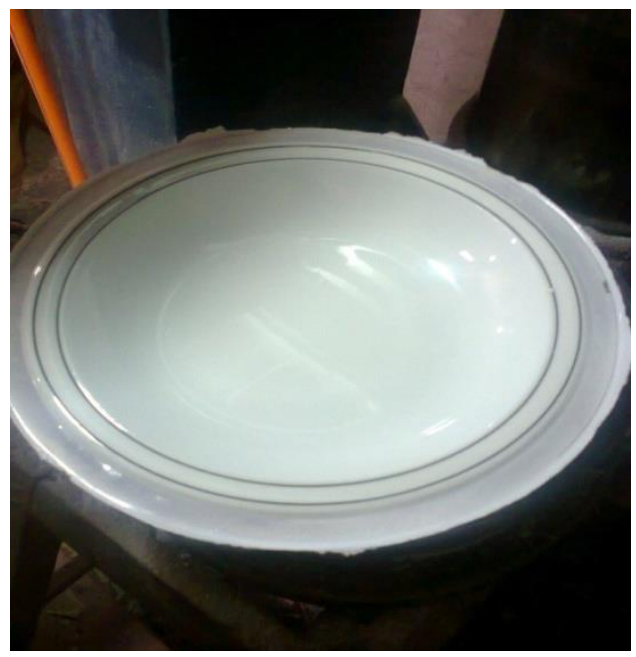

Figure 7

Final Product

The previously mentioned process is totally human dependent and it requires complete attention of the worker especially at two crucial moments described above. A little bit negligence of the worker can spoil the item that will cause financial loss. At the same time for the worker, to ensure a complete attentiveness, it results in a continuous mental stress

\section{PROPOSED EQUIPMENT}

\subsection{Wireless Sensor for Crockery Manufacturing}

Measurement Specialties M7100 Industrial Pressure Transducers

Measurement Specialties M7100 industrial pressure transducer from the Microfused ${ }^{\mathrm{TM}}$ line of MEAS fromTE Connectivity is able to measure liquid or gas pressure, even for media such as contaminated water, steam and corrosive fluids. The transducer pressure cavity is machined from a solid piece of $17-4 \mathrm{PH}$ stainless 
steel. The standard version includes a 1/4 NPT pipe thread allowing a leak-proof, all metal sealed system. Durability is high due to the fact that no O-rings or organics are exposed to the pressure media. This automotive grade pressure transducer with stainless steel hermetic pressure ports and integral electrical connector boasts up to 43,000psi (3000Bar). The M7100 exceeds industrial CE requirements including surge protection and is overvoltage protected to $16 \mathrm{Vdc}$ in both positive and reverse polarity.

\section{FEATURES}

Hermetic Pressure Ports

Integral Electrical Connector

Survives High Vibration 0.25\% Accuracy

Water Resistant $1 \mathrm{M}$ immersion

\section{APPLICATIONS}

HVAC Refrigeration Controls

Off Road Vehicle Engine Control

Compressors

Hydraulics

Energy and Water Management

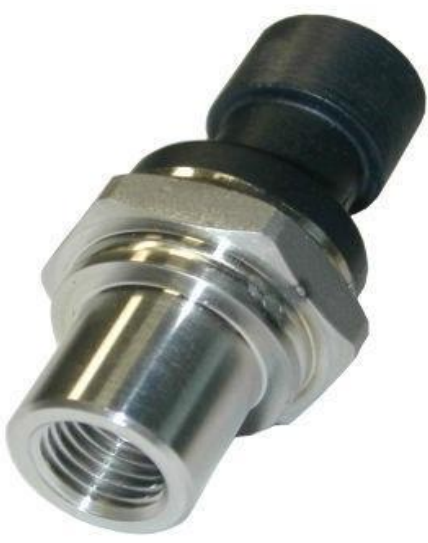

\section{Dimensions}

DIMENSIDN ARE IN INCHES [nm]
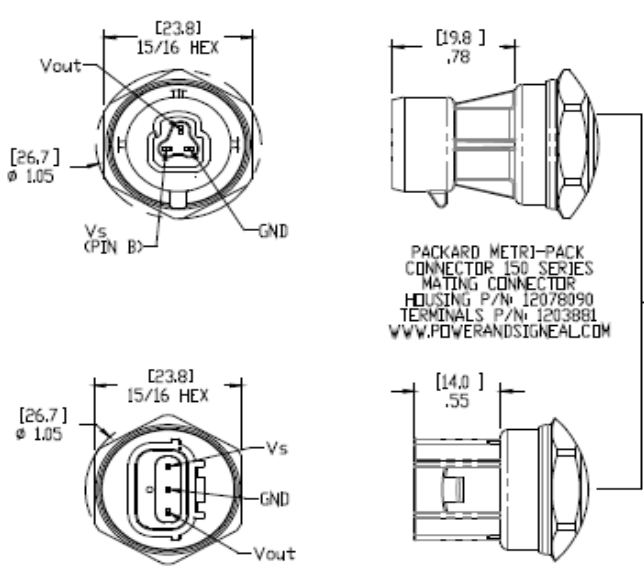

SUMITIMU CDNNECTD

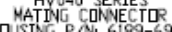

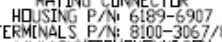

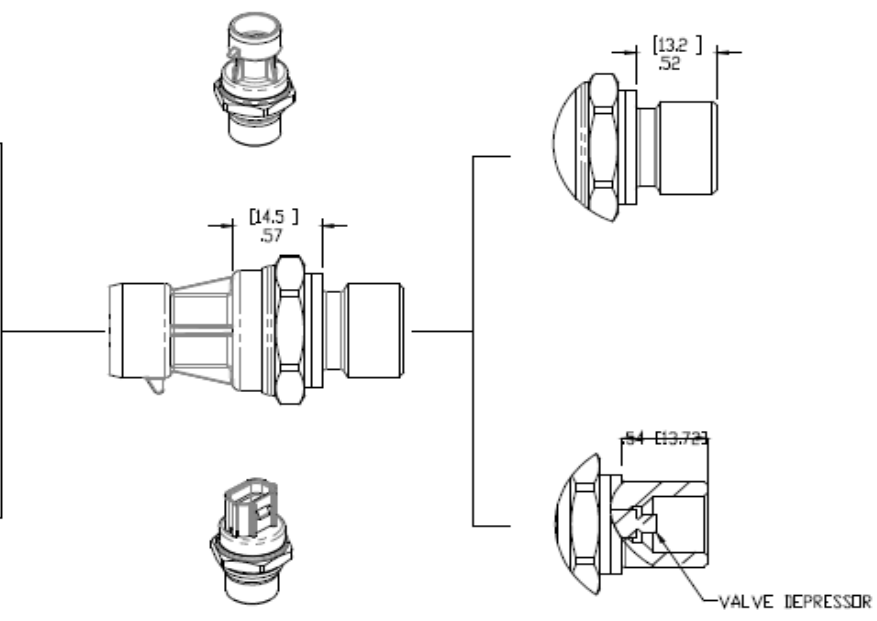




\subsection{Electric Motor}

High Torque Encoder DC Servo Motor 10RPM with Step/Dir Drive

This Encoder DC Servo motor solution integrates an $0.2 \mathrm{deg}$ resolution optical encoder and a high power electronic servo drive on an Industrial grade high torque dc motor. It supports STEP/PULSE and DIRECTION digital inputs that are opto-isolated. This solution works extremely well for slow speeds by providing high correction torque through a closed PI control loop.

The Motor is a Industrial grade 10RPM high torque motor with a massive torque of $120 \mathrm{kgcm}$ in small size. The motor has a metal gearbox with all high quality metal gears and has a off-centered shaft.

\subsection{Wireless Micro Controller}

TI's SimpleLink ${ }^{\text {TM }}$ CC $3 x$ family of wireless MCUs offers the next generation of Tl's embedded $\mathrm{Wi}-\mathrm{Fi} \circledast$ that enables easier development of Internet of Things designs. This Internet-on-a-chip ${ }^{\mathrm{TM}}$ portfolio is based on the industry's first user-dedicated Wi-Fi microcontroller. It supports the WI-FI CERTIFIED ${ }^{\mathrm{TM}}$ standard, designed for easy integration of sensors, and provides low-power consumption for batteryoperated applications.

\section{WIRELESS SENSOR PROTOCOL}

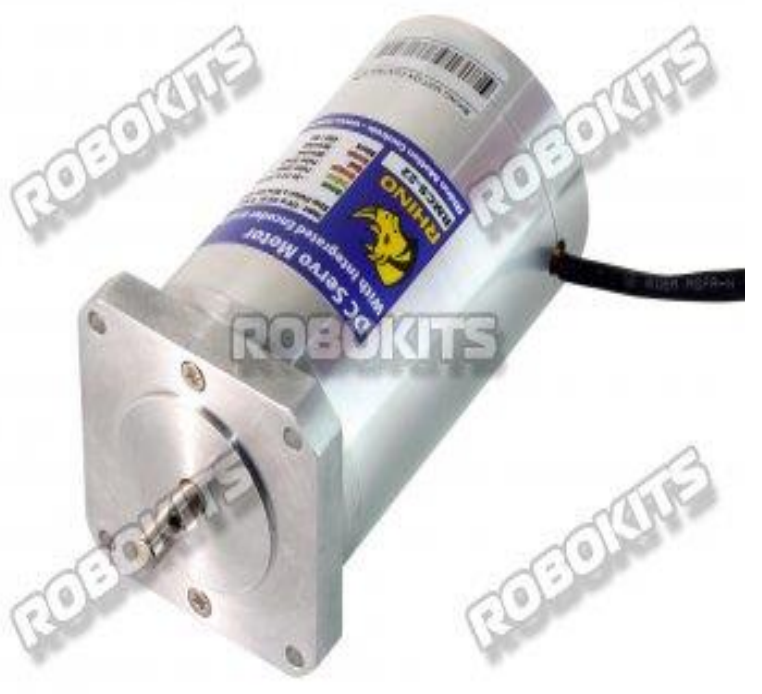

Currently, sensor networks are widely gaining ground because of its numerous applications ranging from environment monitoring, industrial machine and home appliances monitoring. Such network is best described as a network that consists of sensor nodes assigned for a specific function[3]. The sensor node will have full computational ability for sensing and transmitting data in wireless communications model. Inter-connective protocol is considered suitable for wireless sensor networks. Interconnective protocol is capable of detecting damaged node on time through the help of another node closed to the faulty one. For the purpose of this project, the 802.15.4 and ZigBee standards will be discussed for its peculiarity and reliability.

The 802.15.4 and ZigBee standards are considered as a wireless technology with open global standard, addressing the area of power, cost and high radio frequency [4].

\subsection{Proposed Method}

As described in previous section the main objective of our research is to save the item from overheating. To achieve the objective we have to sense when the material comes out from the spillways and how to open the dyes automatically. In order to open or close the dyes, gear lever must pull to push accordingly. Gear lever has three states in center it is considered to be in neutral state, when it is pushes forward it close the dye and when it pushes backward it open the dyes To move the gear lever back and forth we require an electric motor that can be triggered by central control unit.

For this purpose we need:

1. A wireless sensor to sense the pressure on the spillway

2. A wireless Micro Controller

3. An Electric Motor 
Wireless sensor sense the pressure on the spillway that is caused by the marital that comes out from the spillway.

The electric motor is used to move the gear lever in the above stated three states. The proposed electric motor can move at $0^{\circ}, 90^{\circ}$ and $180^{\circ}$. At $90^{\circ}$ the machine is in nutrual state. At $0^{\circ}$ machine is considered in a state that can open the dyes and $180^{\circ}$ is the state to close the dyes.

Wireless Micro Controller is used to trigger and control the movement of the electric motor that results in the movement of the gear lever to open and close the dyes.

\subsection{Working}

The lower part of the dye is filled with raw material and with the help of hydraulic pressure both the part i.e. lower part and upper part of the dyes joins together. When the material is heated it is converted from powder to solid form and takes the shape of dye. A little amount of solid material's waste comes out from the spill way that is pointed above in the fig.
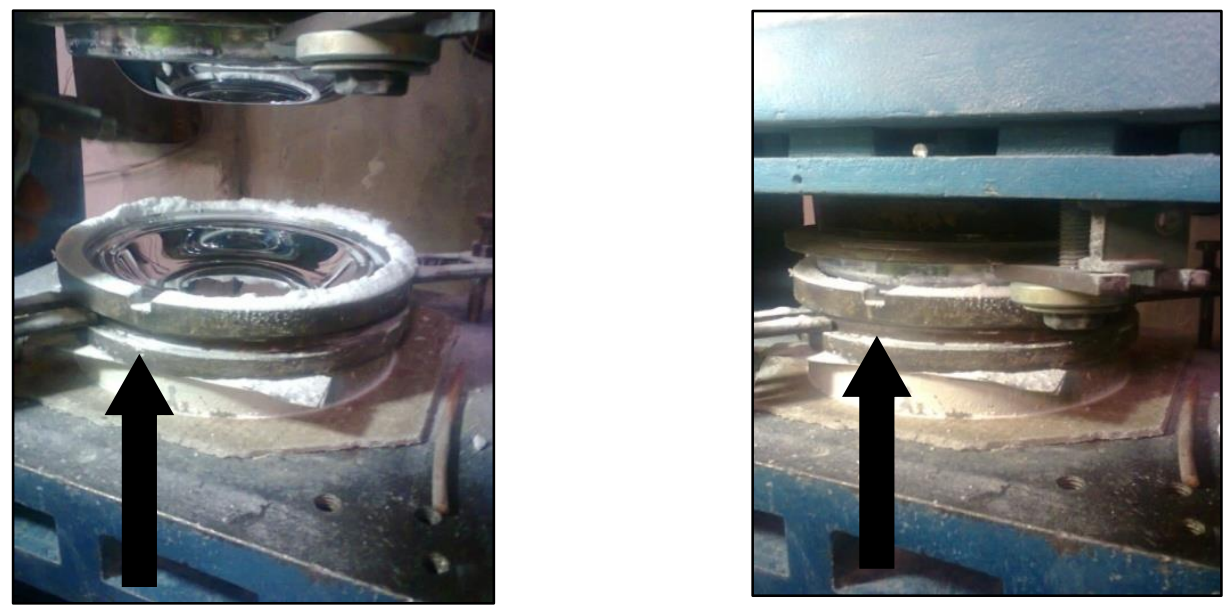

As soon as the waste comes out, the pressure sensor senses the pressure that is placed at spillway, and sends signal to the controlling node. The controlling node sends this information to the central control unit that senses the node which signaled because each node assigned a unique name. Now the central unit triggered the respective micro controller that instantly open and close the dyes to release the air between the dyes that can cause a bubble in the item, now a timer starts in the control unit, after the set time (in second, aprox 8-10 seconds) the control unit again triggered the Micro Controller to move the electric motor in $0^{\circ}$ state that cause to open the dyes and a timer2 starts. Now the worker place the foil of overlay with partially cured piece. After timer2 reaches at a specific set time, the central control unit again triggered the Micro Controller to move the electric motor at $180^{\circ}$ in order to close the dyes.

Again it is heated at specific temperature until the material again comes out. As previous, the sensor sense the pressure at spillways and send information to the central control unit via controlling node which in turn again triggered the Micro Controller to open the dye i.e. move the electric motor in $0^{\circ}$. Hence the process completes and worker takes out the item from dyes.

In this we make improvement in the crockery manufacturing process and reduce the chances of financial loss to zero, that can occurs if the item spoil due to overheating. Also at the same time another benefit of using this technology is that we provide ease for the worker in the form of reducing his mental stress because without using this technology the worker has to be attentive all the time and has to point his eyes on the spill way all the time, to open the part of dyes immediately as soon as waste comes out, in order to save the item from being spoiled.

\section{FINANCIAL BENEFITS}

The author visited some crockery manufacturing unit and finds that daily 5-7 pieces are spoiled due to overheating.

$$
\begin{array}{ll}
1 \text { piece cost } & =200 \text { aprox } \\
5 \text { pieces cost } & =200 \times 5=1000
\end{array}
$$

Annual loss due to overheating $=1000 \times 365=3,65,000$

(only one manufacturing machine) 
Most of the manufacturing units have as low as approximately 10 manufacturing machines. So the annual loss of a Manufacturing unit of 10 machines:

Annual loss of a manufacturing unit (10 machines) $\quad=365000 \times 10=36,50,000$

Which is a significant saving due to the use of proposed methodology, as depicted in graph

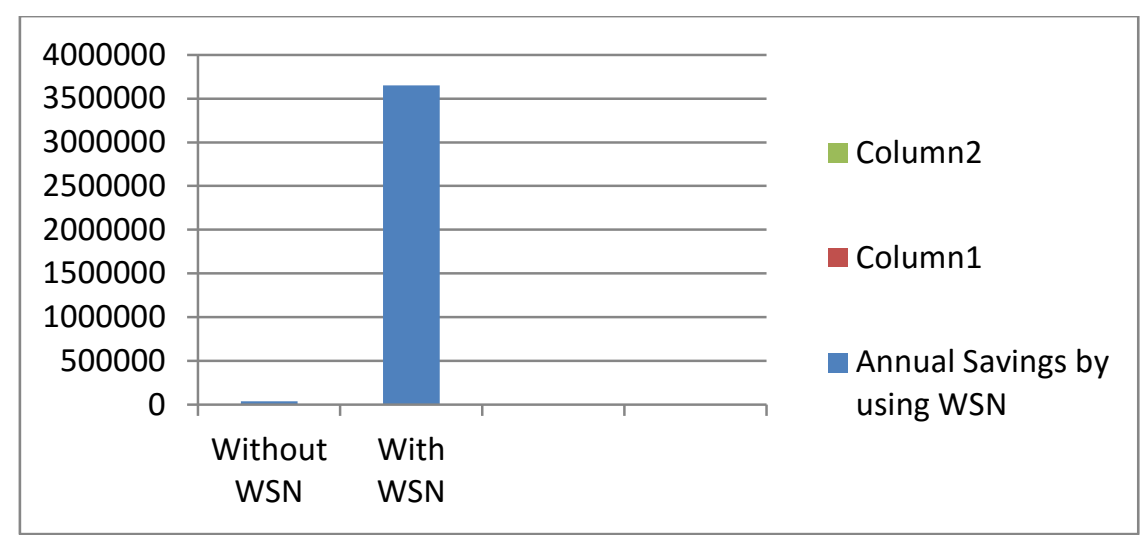

\section{CONCLUSION AND FUTURE DIRECTIONS}

WSN is a technology with promising future and it is presently used in a wide range of applications to offer significant advantages over current system. The methodology explained in the paper provides significant financial benefits as well as it provides mental relief to the worker. Another benefit of using WSN in this industry is that the owner of the manufacturing unit has no need to worry about in finding an experience and efficient worker because all the crucial moments are handled by WSN. The research can be extended to make this production process completely automatic i.e. without human interference.

\section{REFERENCES LIST}

[1] B. Warneke and K. Pister, "MEMS for distributed wireless sensor networks," in Proceedings of the 9th International Conference on Electronics, Circuits and Systems, vol. 1, pp. 291-294,2002.

[2] I. Khemapech, I. Duncan, and A. Miller, "A survey of wireless sensor networks technology," in Proceedings of the 6th Annual Postgraduate Symposium on the Convergence of Telecommunications, Networking and Broadcasting, 2005.

[3] B. Sheng, C. C. Tan, L. Qun, W. Mao, "An Approximation Algorithm for Data Storage Placement in Sensor Networks", International Conference on Wireless Algorithms, Systems and Applications,WASA,pp.71-78,2007.

[4] G. Dawud, M. Beigl, "A Study on the Use of Wireless Sensor Networks in a Retail Store," Distributed and Ubiquitous Computing Institute for Operating Systems and Computer Networks Technical University of Braunschweig,Germany,pp.1-3, 2009.

[5] L. Krishnamurthy, R. Adler, P. Buonadonna, J. Chhabra, M. Flanigan, N. Kushalnagar, L. Nachman, and M. Yarvis. Design and deployment of industrial sensor networks: Experiences from a semiconductor plant and the north sea. In SenSys '05: 3rd International Conference on Embedded Networked Sensor Systems, pages 64-75. ACM, November 2005.

[6] F. Murphy, D. Laffey, B. O'Flynn, J. Buckley, and J. Barton. Development of a wireless sensor network for collaborative agents to treat scale formation in oil pipes. In EWSN, pages 179-194, 2007

[7] I. Stoianov, L. Nachman, S. Madden, and T. Tokmouline. Pipeneta wireless sensor network for pipeline monitoring. In IPSN, pages 264-273, 2007.

[8] A. Tiwari, F.L. Lewis, and S.S. Ge. Wireless sensor network for machine condition based maintenance. In 
Control, Automation, Robotics and Vision Conference, 2004. ICARCV 2004 8th, volume 1, pages 461 -467 Vol. 1, dec. 2004.

[9] P. Wright, D. Dornfeld, and N. Ota. Condition monitoring in end-milling using wireless sensor networks. Transactions of NAMRI/SME, 36, 2008. 